\title{
General iterative methods for generalized equilibrium problems and fixed point problems of $k$-strict pseudo-contractions
}

\section{Dao-Jun Wen* and Yi-An Chen}

\section{"Correspondence:}

daojunwen@163.com

College of Mathematics and

Statistics, Chongqing Technology

and Business University, Chongqing, 400067, China

\begin{abstract}
In this paper, we modify the general iterative method to approximate a common element of the set of solutions of generalized equilibrium problems and the set of common fixed points of a finite family of $k$-strictly pseudo-contractive nonself mappings. Strong convergence theorems are established under some suitable conditions in a real Hilbert space, which also solves some variation inequality problems. Results presented in this paper may be viewed as a refinement and important generalizations of the previously known results announced by many other authors.

MSC: $47 \mathrm{H} 05 ; 47 \mathrm{H} 09 ; 47 \mathrm{H} 10$

Keywords: generalized equilibrium problem; $k$-strict pseudo-contractions; general iterative method; $\alpha$-inverse strongly monotone; common fixed point; strong convergence
\end{abstract}

\section{Introduction}

Let $H$ be a real Hilbert space with inner product $\langle\cdot, \cdot\rangle$ and norm $\|\cdot\|$, respectively. Let $K$ be a nonempty closed convex subset of $H$. Let $A: K \rightarrow H$ be a nonlinear mapping and $F: K \times K \rightarrow \mathbb{R}$ be a bi-function, where $\mathbb{R}$ denotes the set of real numbers. We consider the following generalized equilibrium problem: Find $x \in K$ such that

$$
F(x, y)+\langle A x, y-x\rangle \geq 0, \quad \forall y \in K .
$$

We use $E P(F, A)$ to denote the solution set of the problem (1.1). If $A \equiv 0$, the zero mapping, then the problem (1.1) is reduced to the normal equilibrium problem: Find $x \in K$ such that

$$
F(x, y) \geq 0, \quad \forall y \in K .
$$

We use $E P(F)$ to denote the solution set of the problem (1.2). If $F \equiv 0$, then the problem (1.1) is reduced to the classical variational inequality problem: Find $x \in K$ such that

$$
\langle A x, y-x\rangle \geq 0, \quad \forall y \in K .
$$

๑ 2012 Wen and Chen; licensee Springer. This is an Open Access article distributed under the terms of the Creative Commons Attribution License (http://creativecommons.org/licenses/by/2.0), which permits unrestricted use, distribution, and reproduction in any medium, provided the original work is properly cited. 
The generalized equilibrium problem (1.1) is very general in the sense that it includes, as special cases, optimization problems, variational inequalities, mini-max problems, the Nash equilibrium problem in noncooperative games and others (see, e.g., [1-3]).

Recall that a nonself mapping $T: K \rightarrow H$ is called a $k$-strict pseudo-contraction if there exists a constant $k \in[0,1)$ such that

$$
\|T x-T y\|^{2} \leq\|x-y\|^{2}+k\|(I-T) x-(I-T) y\|^{2}, \quad \forall x, y \in K .
$$

We use $F(T)$ to denote the fixed point set of $T$, i.e., $F(T):=\{x \in K: T x=x\}$. As $k=0, T$ is said to be nonexpansive, i.e.,

$$
\|T x-T y\| \leq\|x-y\|, \quad \forall x, y \in K .
$$

$T$ is said to be pseudo-contractive if $k=1$, and is also said to be strongly pseudo-contractive if there exists a positive constant $\lambda \in(0,1)$ such that $T+\lambda I$ is pseudo-contractive. Clearly, the class of $k$-strict pseudo-contractions falls into the one between classes of nonexpansive mappings and pseudo-contractions. We remark also that the class of strongly pseudocontractive mappings is independent of the class of $k$-strict pseudo-contractions (see, e.g., $[4,5])$.

Iterative methods for equilibrium problems and fixed point problems of nonexpansive mappings have been extensively investigated. However, iterative schemes for strict pseudo-contractions are far less developed than those for nonexpansive mappings though Browder and Petryshyn [5] initiated their work in 1967; the reason is probably that the second term appearing in the right-hand side of (1.3) impedes the convergence analysis for iterative algorithms used to find a fixed point of the strict pseudo-contraction. On the other hand, strict pseudo-contractions have more powerful applications than nonexpansive mappings do in solving inverse problems; see, e.g., $[6-18,20-27]$ and the references therein. Therefore it is interesting to develop the effective iterative methods for equilibrium problems and fixed point problems of strict pseudo-contractions.

In 2006, Marino and $\mathrm{Xu}$ [8] introduced a general iterative method and proved that for a given $x_{0} \in H$, the sequence $\left\{x_{n}\right\}$ generated by

$$
x_{n+1}=\alpha_{n} \gamma f\left(x_{n}\right)+\left(I-\alpha_{n} B\right) T x_{n}, \quad \forall n \in N,
$$

where $T$ is a self-nonexpansive mapping on $H, f$ is a contraction of $H$ into itself and $\left\{\alpha_{n}\right\} \subseteq$ $(0,1)$ satisfies certain conditions, $B$ is a strongly positive bounded linear operator on $H$, converges strongly to $x^{*} \in F(T)$, which is the unique solution of the following variational inequality:

$$
\left\langle(B-\gamma f) x^{*}, x^{*}-x\right\rangle \leq 0, \quad \forall x \in F(T),
$$

and is also the optimality condition for some minimization problem.

Recently, Takahashi and Takahashi [12] considered the equilibrium problem and nonexpansive mapping by viscosity approximation methods. To be more precise, they proved the following theorem. 
Theorem of TT Let $K$ be a nonempty closed convex subset of $H$. Let $F$ be a bi-function from $K \times K$ to $\mathbb{R}$ satisfying (A1)-(A4) and let $T: K \rightarrow H$ be a nonexpansive mapping such that $F(T) \cap E P(F) \neq \phi$. Let $f: H \rightarrow H$ be a contraction and let $\left\{x_{n}\right\}$ and $\left\{y_{n}\right\}$ be sequences generated by $x_{1} \in H$ and

$$
\left\{\begin{array}{l}
F\left(y_{n}, z\right)+\frac{1}{r_{n}}\left\langle z-y_{n}, y_{n}-x_{n}\right\rangle \geq 0, \quad \forall z \in K \\
x_{n+1}=\alpha_{n} f\left(x_{n}\right)+\left(1-\alpha_{n}\right) T y_{n}, \quad n \geq 1
\end{array}\right.
$$

where $\left\{\alpha_{n}\right\} \subset[0,1]$ and $\left\{r_{n}\right\}$ satisfy

$$
\begin{array}{ll}
\lim _{n \rightarrow \infty} \alpha_{n}=0, & \sum_{n=0}^{\infty} \alpha_{n}=\infty, \quad \sum_{n=0}^{\infty}\left|\alpha_{n+1}-\alpha_{n}\right|<\infty, \\
\liminf _{n \rightarrow \infty} r_{n}>0, & \sum_{n=0}^{\infty}\left|r_{n+1}-r_{n}\right|<\infty .
\end{array}
$$

Then $\left\{x_{n}\right\}$ and $\left\{y_{n}\right\}$ converge strongly to $q \in F(T) \cap E P(F)$, where $q=P_{F(T) \cap E P(F)} f(q)$.

In 2009, Ceng et al. [15] further studied the equilibrium problem and fixed point problems of strict pseudo-contraction mappings $T$ by an iterative scheme for finding an element of $E P(F) \cap F(T)$. Very recently, by using the general iterative method Liu [16] proposed the implicit and explicit iterative processes for finding an element of $E P(F) \cap F(T)$ and then obtained some strong convergence theorems, respectively. On the other hand, Takahashi and Takahashi [18] considered the generalized equilibrium problem and nonexpansive mapping in a Hilbert space. Moreover, they constructed an iterative scheme for finding an element of $E P(F, A) \cap F(T)$ and then proved a strong convergence of the iterative sequence under some suitable conditions.

In this paper, inspired and motivated by research going on in this area, we introduce a general iterative method for generalized equilibrium problems and strict pseudocontractive nonself mappings, which is defined in the following way:

$$
\left\{\begin{array}{l}
F\left(u_{n}, y\right)+\left\langle A x_{n}, y-u_{n}\right\rangle+\frac{1}{r_{n}}\left\langle y-u_{n}, u_{n}-x_{n}\right\rangle \geq 0, \quad \forall y \in K \\
y_{n}=\beta_{n} u_{n}+\left(1-\beta_{n}\right) \sum_{i=1}^{N} \eta_{i}^{(n)} T_{i} u_{n}, \\
x_{n+1}=\alpha_{n} \gamma f\left(x_{n}\right)+\left(I-\alpha_{n} B\right) y_{n}, \quad n \geq 1,
\end{array}\right.
$$

where constant $\gamma>0, f$ is a contraction and $A, B$ are two operators, $\left\{T_{i}\right\}_{i=i}^{N}: K \rightarrow H$ is a finite family of $k_{i}$-strict pseudo-contractions, $\left\{\eta_{i}^{(n)}\right\}_{i=1}^{N}$ is a finite sequence of positive numbers, $\left\{\alpha_{n}\right\},\left\{\beta_{n}\right\}$ and $\left\{r_{n}\right\}$ are some sequences with certain conditions.

Our purpose is not only to modify the general iterative method to the case of a finite family of $k_{i}$-strictly pseudo-contractive nonself mappings, but also to establish strong convergence theorems for a generalized equilibrium problem and $k_{i}$-strict pseudo-contractions in a real Hilbert space, which also solves some variation inequality problems. Our theorems presented in this paper improve and extend the corresponding results of $[12,15,16$, $18,20,21,25]$. 


\section{Preliminaries}

Let $K$ be a nonempty closed convex subset of a real Hilbert $H$ space with inner product $\langle\cdot, \cdot\rangle$ and norm $\|\cdot\|$, respectively. Recall that a mapping $f: K \rightarrow K$ is a contraction, if there exists a constant $\rho \in(0,1)$ such that

$$
\|f(x)-f(y)\| \leq \rho\|x-y\|, \quad \forall x, y \in K .
$$

We use $\Pi_{K}$ to denote the collection of all contractions on $K$. The operator $A: K \rightarrow H$ is said to be monotone if

$$
\langle A x-A y, x-y\rangle \geq 0, \quad \forall x, y \in K .
$$

$A: K \rightarrow H$ is said to be $r$-strongly monotone if there exists a constant $r>0$ such that

$$
\langle A x-A y, x-y\rangle \geq r\|x-y\|^{2}, \quad \forall x, y \in K .
$$

$A: K \rightarrow H$ is said to be $\alpha$-inverse strongly monotone if there exists a constant $\alpha>0$ such that

$$
\langle A x-A y, x-y\rangle \geq \alpha\|A x-A y\|^{2}, \quad \forall x, y \in K .
$$

Recall that an operator $B$ is strongly positive if there exists a constant $\bar{\gamma}>0$ with the property

$$
\langle B x, x\rangle \geq \bar{\gamma}\|x\|^{2}, \quad \forall x \in H .
$$

To study the generalized equilibrium problem (1.1), we may assume that the bi-function $F: K \times K \rightarrow \mathbb{R}$ satisfies the following conditions:

(A1) $F(x, x)=0$ for all $x \in K$;

(A2) $F$ is monotone, i.e., $F(x, y)+F(y, x) \leq 0$ for all $x, y \in K$;

(A3) for each $x, y, z \in K, \lim _{t \rightarrow 0} F(t z+(1-t) x, y) \leq F(x, y)$;

(A4) for each $x \in K, y \mapsto F(x, y)$ is convex and lower semi-continuous.

In order to prove our main results, we need the following lemmas and propositions.

Lemma 2.1 [1,3] Let $F: K \times K \rightarrow \mathbb{R}$ be a bi-function satisfying (A1)-(A4). Then, for any $r>0$ and $x \in H$, there exists $z \in K$ such that

$$
F(z, y)+\frac{1}{r}\langle y-z, z-x\rangle \geq 0, \quad \forall y \in K .
$$

Further, if $T_{r} x=\left\{z \in K: F(z, y)+\frac{1}{r}\langle y-z, z-x\rangle \geq 0, \forall y \in K\right\}$, then the following hold:

(1) $T_{r}$ is single-valued;

(2) $T_{r}$ is firmly nonexpansive, i.e, $\left\|T_{r} x-T_{r} y\right\|^{2} \leq\left\langle T_{r} x-T_{r} y, x-y\right\rangle$ for all $x, y \in H$;

(3) $F\left(T_{r}\right)=E P(F)$;

(4) $E P(F)$ is closed and convex.

Lemma 2.2 [8] In the Hilbert space H, there hold the following identities: 
(i) $\|x+y\|^{2}=\|x\|^{2}+2\langle x, y\rangle+\|y\|^{2} \leq\|x\|^{2}+2\langle y,(x+y)\rangle, \forall x, y \in H$;

(ii) $\|t x+(1-t) y\|^{2}=t\|x\|^{2}+(1-t)\|y\|^{2}-t(1-t)\|x-y\|^{2}, \forall t \in[0,1], \forall x, y \in H$.

Lemma 2.3 [8] Assume that B is a strongly positive linear bounded operator on the Hilbert space $H$ with a coefficient $\bar{\gamma}>0$ and $0<\varrho<\|B\|^{-1}$. Then $\|I-\varrho B\| \leq 1-\varrho \bar{\gamma}$.

Lemma 2.4 [10] If $T: K \rightarrow H$ is a $k$-strict pseudo-contraction, then the fixed point set $F(T)$ is closed convex so that the projection $P_{F(T)}$ is well defined.

Lemma $2.5[2,10]$ Let $T: K \rightarrow H$ be a $k$-strict pseudo-contraction. For $\lambda \in[k, 1)$, define $S: K \rightarrow H$ by $S x=\lambda x+(1-\lambda) T x$ for each $x \in K$. Then $S$ is a nonexpansive mapping such that $F(S)=F(T)$.

Lemma 2.6 [19] Assume $\left\{a_{n}\right\}$ is a sequence of nonnegative real numbers such that

$$
a_{n+1} \leq\left(1-\gamma_{n}\right) a_{n}+\gamma_{n} \delta_{n}, \quad n \geq 0
$$

where $\left\{\gamma_{n}\right\}$ is a sequence in $(0,1)$ and $\left\{\delta_{n}\right\}$ is a real sequence such that

(i) $\sum_{n=1}^{\infty} \gamma_{n}=\infty$;

(ii) $\lim \sup _{n \rightarrow \infty} \delta_{n} \leq 0$ or $\sum_{n=1}^{\infty}\left|\gamma_{n} \delta_{n}\right|<\infty$.

Then $\lim _{n \rightarrow \infty} a_{n}=0$.

Proposition 2.1 (See, e.g., Acedo and Xu [20]) Let $K$ be a nonempty closed convex subset of the Hilbert space $H$. Given an integer $N \geq 1$, assume that $\left\{T_{i}\right\}_{i=1}^{N}: K \rightarrow H$ is a finite family of $k_{i}$-strict pseudo-contractions. Suppose that $\left\{\lambda_{i}\right\}_{i=1}^{N}$ is a positive sequence such that $\sum_{i=1}^{N} \lambda_{i}=1$. Then $\sum_{i=1}^{N} \lambda_{i} T_{i}$ is a $k$-strict pseudo-contraction with $k=\max \left\{k_{i}: 1 \leq i \leq N\right\}$.

Proposition 2.2 (See, e.g., Acedo and Xu [20]) Let $\left\{T_{i}\right\}_{i=1}^{N}$ and $\left\{\lambda_{i}\right\}_{i=1}^{N}$ be given as in Proposition 2.1 above. Then $F\left(\sum_{i=1}^{N} \lambda_{i} T_{i}\right)=\bigcap_{i=1}^{N} F\left(T_{i}\right)$.

\section{Main results}

Theorem 3.1 Let $K$ be a nonempty closed convex subset of the Hilbert space $H$ and $F$ : $K \times K \rightarrow \mathbb{R}$ be a bi-function satisfying (A1)-(A4). Let $A$ be an $\alpha$-inverse strongly monotone mapping and $B$ be a strongly positive bounded linear operator on $H$ with $\bar{\gamma}>0$. Assume that $\left\{T_{i}\right\}_{i=1}^{N}: K \rightarrow H$ be a finite family of $k_{i}$-strict pseudo-contractions such that $\mathcal{F}=\bigcap_{i=1}^{N} F\left(T_{i}\right) \cap$ $\operatorname{EP}(F, A) \neq \phi$. Suppose $f \in \Pi_{K}$ with a coefficient $\rho \in(0,1)$ and $\left\{\eta_{i}^{(n)}\right\}_{i=1}^{N}$ are finite sequences of positive numbers such that $\sum_{i=1}^{N} \eta_{i}^{(n)}=1$ for all $n \geq 0$, for a given point $x_{0} \in K, \alpha_{n}, \beta_{n} \in(0,1)$, $r_{n} \in(0,2 \alpha)$ and $0<\gamma<\frac{\bar{\gamma}}{\rho}$, the following control conditions are satisfied:

(i) $\lim _{n \rightarrow \infty} \alpha_{n}=0, \sum_{n=1}^{\infty} \alpha_{n}=\infty$ and $\sum_{n=1}^{\infty}\left|\alpha_{n}-\alpha_{n-1}\right|<\infty$;

(ii) $k_{i} \leq \beta_{n} \leq \lambda<1, \lim _{n \rightarrow \infty} \beta_{n}=\lambda$ and $\sum_{n=1}^{\infty}\left|\beta_{n}-\beta_{n-1}\right|<\infty$;

(iii) $\sum_{n=1}^{\infty} \sum_{i=1}^{N}\left|\eta_{i}^{(n)}-\eta_{i}^{(n-1)}\right|<\infty$;

(iv) $\liminf _{n \rightarrow \infty} r_{n}>0$ and $\sum_{n=1}^{\infty}\left|r_{n}-r_{n-1}\right|<\infty$.

Then the sequence $\left\{x_{n}\right\}$ generated by (1.4) converges strongly to $q \in \mathcal{F}$, which solves the variational inequality

$$
\langle(B-\gamma f) q, q-p\rangle \leq 0, \quad \forall p \in \mathcal{F}
$$


Proof Putting $W_{n}=\sum_{i=1}^{N} \eta_{i}^{(n)} T_{i}$, we have $W_{n}: K \rightarrow H$ is a $k$-strict pseudo-contraction and $F\left(W_{n}\right)=\bigcap_{i=1}^{N} F\left(T_{i}\right)$ by Proposition 2.1 and 2.2 , where $k=\max \left\{k_{i}: 1 \leq i \leq N\right\}$.

First, we show that the mapping $I-r_{n} A$ is nonexpansive. Indeed, for each $x, y \in K$, we have

$$
\begin{aligned}
\left\|\left(I-r_{n} A\right) x-\left(I-r_{n} A\right) y\right\|^{2} & =\|x-y\|^{2}-2 r_{n}\langle x-y, A x-A y\rangle+r_{n}^{2}\|A x-A y\|^{2} \\
& \leq\|x-y\|^{2}-2 \alpha r_{n}\|A x-A y\|^{2}+r_{n}^{2}\|A x-A y\|^{2} \\
& =\|x-y\|^{2}-r_{n}\left(2 \alpha-r_{n}\right)\|A x-A y\|^{2} .
\end{aligned}
$$

It follows from the condition $r_{n} \in(0,2 \alpha)$ that the mapping $I-r_{n} A$ is nonexpansive. From Lemma 2.1, we see that $E P(F, A)=F\left(T_{r_{n}}\left(I-r_{n} A\right)\right)$. Note that $u_{n}$ can be rewritten as $u_{n}=$ $T_{r_{n}}\left(I-r_{n} A\right) x_{n}$ and $p=T_{r_{n}}\left(I-r_{n} A\right) p$ for each $n \geq 1$ as $p \in \mathcal{F}$.

From (1.4), condition (ii) and Lemma 2.2, we have

$$
\begin{aligned}
\left\|y_{n}-p\right\|^{2}= & \left\|\beta_{n}\left(u_{n}-p\right)+\left(1-\beta_{n}\right)\left(W_{n} u_{n}-p\right)\right\|^{2} \\
= & \beta_{n}\left\|u_{n}-p\right\|^{2}+\left(1-\beta_{n}\right)\left\|W_{n} u_{n}-p\right\|^{2}-\beta_{n}\left(1-\beta_{n}\right)\left\|u_{n}-W_{n} u_{n}\right\|^{2} \\
\leq & \beta_{n}\left\|u_{n}-p\right\|^{2}+\left(1-\beta_{n}\right)\left[\left\|u_{n}-p\right\|^{2}+k\left\|u_{n}-W_{n} u_{n}\right\|^{2}\right] \\
& -\beta_{n}\left(1-\beta_{n}\right)\left\|u_{n}-W_{n} u_{n}\right\|^{2} \\
= & \left\|u_{n}-p\right\|^{2}-\left(1-\beta_{n}\right)\left(\beta_{n}-k\right)\left\|u_{n}-W_{n} u_{n}\right\|^{2} \\
\leq & \left\|u_{n}-p\right\|^{2} .
\end{aligned}
$$

By $u_{n}=T_{r_{n}}\left(I-r_{n} A\right) x_{n}$, we obtain

$$
\left\|u_{n}-p\right\|=\left\|T_{r_{n}}\left(I-r_{n} A\right) x_{n}-p\right\| \leq\left\|x_{n}-p\right\| .
$$

This together with (3.1), we see that

$$
\left\|y_{n}-p\right\| \leq\left\|u_{n}-p\right\| \leq\left\|x_{n}-p\right\|
$$

Furthermore, by Lemma 2.3, we have

$$
\begin{aligned}
\left\|x_{n+1}-p\right\| & =\left\|\alpha_{n}\left[\gamma f\left(x_{n}\right)-B p\right]+\left(I-\alpha_{n} B\right)\left(y_{n}-p\right)\right\| \\
& \leq\left(1-\alpha_{n} \bar{\gamma}\right)\left\|y_{n}-p\right\|+\alpha_{n}\left\|\gamma f\left(x_{n}\right)-B p\right\| \\
& \leq\left(1-\alpha_{n} \bar{\gamma}\right)\left\|y_{n}-p\right\|+\alpha_{n}\left[\left\|\gamma f\left(x_{n}\right)-\gamma f(p)\right\|+\|\gamma f(p)-B p\|\right] \\
& \leq\left[1-(\bar{\gamma}-\gamma \rho) \alpha_{n}\right]\left\|x_{n}-p\right\|+\alpha_{n}\|\gamma f(p)-B p\| .
\end{aligned}
$$

It follows from induction that

$$
\left\|x_{n}-p\right\| \leq \max \left\{\left\|x_{0}-p\right\|, \frac{1}{\bar{\gamma}-\gamma \rho}\|\gamma f(p)-B p\|\right\}, \quad n \geq 1,
$$

which gives that sequence $\left\{x_{n}\right\}$ is bounded, and so are $\left\{u_{n}\right\}$ and $\left\{y_{n}\right\}$. 
Define a mapping $S_{n} x:=\beta_{n} x+\left(1-\beta_{n}\right) W_{n} x$ for each $x \in K$. Then $S_{n}: K \rightarrow H$ is nonexpansive. Indeed, by using (1.3), Lemma 2.2 and condition (ii), we have for all $x, y \in K$ that

$$
\begin{aligned}
\left\|S_{n} x-S_{n} y\right\|^{2}= & \left\|\beta_{n}(x-y)+\left(1-\beta_{n}\right)\left(W_{n} x-W_{n} y\right)\right\|^{2} \\
= & \beta_{n}\|x-y\|^{2}+\left(1-\beta_{n}\right)\left\|W_{n} x-W_{n} y\right\|^{2} \\
& \quad-\beta_{n}\left(1-\beta_{n}\right)\left\|x-W_{n} x-\left(y-W_{n} y\right)\right\|^{2} \\
\leq & \beta_{n}\|x-y\|^{2}+\left(1-\beta_{n}\right)\left[\|x-y\|^{2}+k\left\|x-W_{n} x-\left(y-W_{n} y\right)\right\|^{2}\right] \\
& \quad-\beta_{n}\left(1-\beta_{n}\right)\left\|x-W_{n} x-\left(y-W_{n} y\right)\right\|^{2} \\
= & \|x-y\|^{2}-\left(1-\beta_{n}\right)\left(\beta_{n}-k\right)\left\|x-W_{n} x-\left(y-W_{n} y\right)\right\|^{2} \\
\leq & \|x-y\|^{2},
\end{aligned}
$$

which shows that $S_{n}: K \rightarrow H$ is nonexpansive.

Next, we show that $\lim _{n \rightarrow \infty}\left\|x_{n+1}-x_{n}\right\|=0$. From (1.4) and Lemma 2.3, we have

$$
\begin{aligned}
\left\|x_{n+1}-x_{n}\right\|= & \left\|\alpha_{n} \gamma f\left(x_{n}\right)+\left(I-\alpha_{n} B\right) y_{n}-\left[\alpha_{n-1} \gamma f\left(x_{n-1}\right)+\left(I-\alpha_{n-1} B\right) y_{n-1}\right]\right\| \\
\leq & \alpha_{n} \gamma\left\|f\left(x_{n}\right)-f\left(x_{n-1}\right)\right\|+\left|\alpha_{n}-\alpha_{n-1}\right|\left[\gamma\left\|f\left(x_{n-1}\right)\right\|+\left\|B y_{n-1}\right\|\right] \\
& +\left\|\left(I-\alpha_{n} B\right)\left(y_{n}-y_{n-1}\right)\right\| \\
\leq & \alpha_{n} \gamma \rho\left\|x_{n}-x_{n-1}\right\|+\left|\alpha_{n}-\alpha_{n-1}\right| M_{1}+\left(1-\alpha_{n} \bar{\gamma}\right)\left\|y_{n}-y_{n-1}\right\|,
\end{aligned}
$$

where $M_{1}=\sup _{n \geq 1}\left\{\gamma\left\|f\left(x_{n}\right)\right\|+\left\|B y_{n}\right\|\right\}<\infty$. Moreover, we note that $y_{n}=S_{n} u_{n}$ and

$$
\begin{aligned}
\left\|y_{n}-y_{n-1}\right\| \leq & \left\|S_{n} u_{n}-S_{n} u_{n-1}\right\|+\left\|S_{n} u_{n-1}-S_{n-1} u_{n-1}\right\| \\
\leq & \left\|u_{n}-u_{n-1}\right\|+\| \beta_{n} u_{n-1}+\left(1-\beta_{n}\right) W_{n} u_{n-1} \\
& \quad-\left[\beta_{n-1} u_{n-1}+\left(1-\beta_{n-1}\right) W_{n-1} u_{n-1}\right] \| \\
\leq & \left\|u_{n}-u_{n-1}\right\|+\left|\beta_{n}-\beta_{n-1}\right|\left\|u_{n-1}-W_{n-1} u_{n-1}\right\| \\
& \quad+\left(1-\beta_{n}\right)\left\|W_{n} u_{n-1}-W_{n-1} u_{n-1}\right\| \\
& \leq\left\|u_{n}-u_{n-1}\right\|+\left|\beta_{n}-\beta_{n-1}\right| M_{2}+\left(1-\beta_{n}\right) \sum_{i=1}^{N}\left|\eta_{i}^{(n)}-\eta_{i}^{(n-1)}\right|\left\|T_{i} u_{n-1}\right\|,
\end{aligned}
$$

where $M_{2}=\sup _{n \geq 1}\left\{\left\|u_{n-1}-W_{n-1} u_{n-1}\right\|\right\}$. On the other hand, we note that

$$
\left\{\begin{array}{l}
F\left(u_{n}, y\right)+\left\langle A x_{n}, y-u_{n}\right\rangle+\frac{1}{r_{n}}\left\langle y-u_{n}, u_{n}-x_{n}\right\rangle \geq 0, \\
F\left(u_{n-1}, y\right)+\left\langle A x_{n-1}, y-u_{n-1}\right\rangle+\frac{1}{r_{n-1}}\left\langle y-u_{n-1}, u_{n-1}-x_{n-1}\right\rangle \geq 0 .
\end{array}\right.
$$

Putting $y=u_{n-1}$ and $y=u_{n}$ in (3.6) respectively, we have

$$
\left\{\begin{array}{l}
F\left(u_{n}, u_{n-1}\right)+\left\langle A x_{n}, u_{n-1}-u_{n}\right\rangle+\frac{1}{r_{n}}\left\langle u_{n-1}-u_{n}, u_{n}-x_{n}\right\rangle \geq 0 \\
F\left(u_{n-1}, u_{n}\right)+\left\langle A x_{n-1}, u_{n}-u_{n-1}\right\rangle+\frac{1}{r_{n-1}}\left\langle u_{n}-u_{n-1}, u_{n-1}-x_{n-1}\right\rangle \geq 0
\end{array}\right.
$$


It follows from (A2) that

$$
\left\langle u_{n}-u_{n-1}, \frac{u_{n-1}-\left(I-r_{n-1} A\right) x_{n-1}}{r_{n-1}}-\frac{u_{n}-\left(I-r_{n} A\right) x_{n}}{r_{n}}\right\rangle \geq 0,
$$

and hence

$$
\left\langle u_{n}-u_{n-1}, u_{n-1}-u_{n}+u_{n}-\left(I-r_{n-1} A\right) x_{n-1}-\frac{r_{n-1}}{r_{n}}\left[u_{n}-\left(I-r_{n} A\right) x_{n}\right]\right\rangle \geq 0 .
$$

Since $\lim _{n \rightarrow \infty} r_{n}>0$, we assume that there exists a real number $\mu$ such that $r_{n}>\mu>0$ for all $n \in N$. Consequently, we have

$$
\begin{aligned}
\left\|u_{n}-u_{n-1}\right\|^{2} \leq & \left\langle u_{n}-u_{n-1},\left(I-r_{n} A\right) x_{n}-\left(I-r_{n-1} A\right) x_{n-1}\right. \\
& \left.+\left(1-\frac{r_{n-1}}{r_{n}}\right)\left[u_{n}-\left(I-r_{n} A\right) x_{n}\right]\right\rangle \\
\leq & \left\|u_{n}-u_{n-1}\right\|\left[\left\|x_{n}-x_{n-1}\right\|+\left|r_{n}-r_{n-1}\right|\left\|A x_{n-1}\right\|\right. \\
& \left.+\frac{r_{n}-r_{n-1}}{r_{n}}\left\|u_{n}-\left(I-r_{n} A\right) x_{n}\right\|\right]
\end{aligned}
$$

and hence

$$
\begin{aligned}
\left\|u_{n}-u_{n-1}\right\| & \leq\left\|x_{n}-x_{n-1}\right\|+\left|r_{n}-r_{n-1}\right|\left\|A x_{n-1}\right\|+\frac{r_{n}-r_{n-1}}{r_{n}}\left\|u_{n}-\left(I-r_{n} A\right) x_{n}\right\| \\
& \leq\left\|x_{n}-x_{n-1}\right\|+\left|r_{n}-r_{n-1}\right|\left[\left\|A x_{n-1}\right\|+\frac{1}{\mu}\left\|u_{n}-\left(I-r_{n} A\right) x_{n}\right\|\right] \\
& \leq\left\|x_{n}-x_{n-1}\right\|+\left|r_{n}-r_{n-1}\right| M_{3},
\end{aligned}
$$

where $M_{3}=\sup \left\{\left\|A x_{n-1}\right\|+\frac{1}{\mu}\left\|u_{n}-\left(I-r_{n} A\right) x_{n}\right\|, n \in N\right\}$. Combining (3.4), (3.5) and (3.8), we have

$$
\begin{aligned}
\left\|x_{n+1}-x_{n}\right\| \leq & \alpha_{n} \gamma \rho\left\|x_{n}-x_{n-1}\right\|+\left|\alpha_{n}-\alpha_{n-1}\right| M_{1}+\left(1-\alpha_{n} \bar{\gamma}\right)\left[\left\|x_{n}-x_{n-1}\right\|\right. \\
& \left.+\left|\beta_{n}-\beta_{n-1}\right| M_{2}+\left|r_{n}-r_{n-1}\right| M_{3}+\left(1-\beta_{n}\right) \sum_{i=1}^{N}\left|\eta_{i}^{(n)}-\eta_{i}^{(n-1)}\right|\left\|T_{i} u_{n-1}\right\|\right] \\
\leq & {\left[1-(\bar{\gamma}-\gamma \rho) \alpha_{n}\right]\left\|x_{n}-x_{n-1}\right\|+\left|\alpha_{n}-\alpha_{n-1}\right| M_{1}+\left|\beta_{n}-\beta_{n-1}\right| M_{2} } \\
& +\left|r_{n}-r_{n-1}\right| M_{3}+\sum_{i=1}^{N}\left|\eta_{i}^{(n)}-\eta_{i}^{(n-1)}\right|\left\|T_{i} u_{n-1}\right\| .
\end{aligned}
$$

It follows from $0<\gamma<\frac{\bar{\gamma}}{\rho}$ and Lemma 2.6 that

$$
\lim _{n \rightarrow \infty}\left\|x_{n+1}-x_{n}\right\|=0
$$

Moreover, we observe that

$$
\left\|x_{n}-y_{n}\right\| \leq\left\|x_{n}-x_{n+1}\right\|+\left\|x_{n+1}-y_{n}\right\| \leq\left\|x_{n}-x_{n+1}\right\|+\alpha_{n}\left\|\gamma f\left(x_{n}\right)-B y_{n}\right\| .
$$


It follows from $\lim _{n \rightarrow \infty} \alpha_{n}=0$ and (3.9) that

$$
\lim _{n \rightarrow \infty}\left\|x_{n}-y_{n}\right\|=0 \text {. }
$$

For $p \in F\left(S_{n}\right) \cap E P(F, A)$, we note that $u_{n}=T_{r_{n}}\left(I-r_{n} A\right) x_{n}$ and

$$
\begin{aligned}
\left\|u_{n}-p\right\|^{2} & =\left\|T_{r_{n}}\left(I-r_{n} A\right) x_{n}-T_{r_{n}}\left(I-r_{n} A\right) p\right\|^{2} \leq\left\langle x_{n}-p, u_{n}-p\right\rangle \\
& =\frac{1}{2}\left(\left\|x_{n}-p\right\|^{2}+\left\|u_{n}-p\right\|^{2}-\left\|x_{n}-u_{n}\right\|^{2}\right),
\end{aligned}
$$

which implies that

$$
\left\|u_{n}-p\right\|^{2} \leq\left\|x_{n}-p\right\|^{2}-\left\|x_{n}-u_{n}\right\|^{2} .
$$

From (1.4), (3.2) and (3.11), we have

$$
\begin{aligned}
\left\|x_{n+1}-p\right\|^{2}= & \left\|\alpha_{n}\left[\gamma f\left(x_{n}\right)-B p\right]+\left(I-\alpha_{n} B\right)\left(y_{n}-p\right)\right\|^{2} \\
\leq & \left(1-\alpha_{n} \bar{\gamma}\right)^{2}\left\|y_{n}-p\right\|^{2}+\alpha_{n}^{2}\left\|\gamma f\left(x_{n}\right)-B p\right\|^{2} \\
& +2 \alpha_{n}\left(1-\alpha_{n} \bar{\gamma}\right)\left\|\gamma f\left(x_{n}\right)-B p\right\|\left\|y_{n}-p\right\| \\
\leq & \left\|u_{n}-p\right\|^{2}+\alpha_{n}^{2}\left\|\gamma f\left(x_{n}\right)-B p\right\|^{2}+2 \alpha_{n}\left\|\gamma f\left(x_{n}\right)-B p\right\|\left\|y_{n}-p\right\| \\
\leq & \left\|x_{n}-p\right\|^{2}-\left\|x_{n}-u_{n}\right\|^{2}+\alpha_{n}^{2}\left\|\gamma f\left(x_{n}\right)-B p\right\|^{2} \\
& +2 \alpha_{n}\left\|\gamma f\left(x_{n}\right)-B p\right\|\left\|y_{n}-p\right\| .
\end{aligned}
$$

Using $\lim _{n \rightarrow \infty} \alpha_{n}=0$ and (3.9) again, we obtain

$$
\lim _{n \rightarrow \infty}\left\|x_{n}-u_{n}\right\|=\lim _{n \rightarrow \infty}\left\|x_{n}-T_{r_{n}}\left(I-r_{n} A\right) x_{n}\right\|=0 .
$$

By the nonexpansion of $S_{n}$, we have

$$
\begin{aligned}
\left\|x_{n}-S_{n} x_{n}\right\| & \leq\left\|x_{n}-x_{n+1}\right\|+\left\|x_{n+1}-S_{n} x_{n}\right\| \\
& \leq\left\|x_{n}-x_{n+1}\right\|+\left\|\alpha_{n} \gamma f\left(x_{n}\right)+\left(I-\alpha_{n} B\right) y_{n}-S_{n} x_{n}\right\| \\
& \leq\left\|x_{n}-x_{n+1}\right\|+\alpha_{n}\left[\left\|\gamma f\left(x_{n}\right)\right\|+\left\|B y_{n}\right\|\right]+\left\|S_{n} u_{n}-S_{n} x_{n}\right\| \\
& \leq\left\|x_{n}-x_{n+1}\right\|+\alpha_{n}\left[\left\|\gamma f\left(x_{n}\right)\right\|+\left\|B y_{n}\right\|\right]+\left\|u_{n}-x_{n}\right\| .
\end{aligned}
$$

This together with (3.9) and (3.12), we obtain

$$
\lim _{n \rightarrow \infty}\left\|x_{n}-S_{n} x_{n}\right\|=0
$$

Furthermore, we note that

$$
\left\|x_{n}-S_{n} x_{n}\right\|=\left(1-\beta_{n}\right)\left\|x_{n}-W_{n} x_{n}\right\| .
$$

It follows from condition (ii) that

$$
\lim _{n \rightarrow \infty}\left\|x_{n}-W_{n} x_{n}\right\|=0 .
$$


On the other hand, by condition (iii), we may assume that $\eta_{i}^{(n)} \rightarrow \eta_{i}$ as $n \rightarrow \infty$ for every $1 \leq i \leq N$. It is easily seen that each $\eta_{i}>0$ and $\sum_{i=1}^{N} \eta_{i}=1$. Define $W=\sum_{i=1}^{N} \eta_{i} T_{i}$, then $W: K \rightarrow H$ is a $k$-strict pseudo-contraction such that $F(W)=F\left(W_{n}\right)=\bigcap_{i=1}^{N} F\left(T_{i}\right)$ by Proposition 2.1 and 2.2. Consequently,

$$
\begin{aligned}
\left\|x_{n}-W x_{n}\right\| & \leq\left\|x_{n}-W_{n} x_{n}\right\|+\left\|W_{n} x_{n}-W x_{n}\right\| \\
& \leq\left\|x_{n}-W_{n} x_{n}\right\|+\sum_{i=1}^{N}\left|\eta_{i}^{(n)}-\eta_{i}\right|\left\|T_{i} x_{n}\right\|,
\end{aligned}
$$

which implies that

$$
\lim _{n \rightarrow \infty}\left\|x_{n}-W x_{n}\right\|=0
$$

Combining (3.14) and (3.15), we obtain

$$
\lim _{n \rightarrow \infty}\left\|W_{n} x_{n}-W x_{n}\right\|=0
$$

Define $S: K \rightarrow H$ by $S x=\lambda x+(1-\lambda) W x$. By condition (ii) again, we have $\lim _{n \rightarrow \infty} \beta_{n}=\lambda \in$ $[k, 1)$. Then, $S$ is nonexpansive with $F(S)=F(W)$ by Lemma 2.5 . Notice that

$$
\begin{aligned}
\left\|x_{n}-S x_{n}\right\| & \leq\left\|x_{n}-S_{n} x_{n}\right\|+\left\|S_{n} x_{n}-S x_{n}\right\| \\
& =\left\|x_{n}-S_{n} x_{n}\right\|+\left\|\beta_{n} x_{n}+\left(1-\beta_{n}\right) W_{n} x_{n}-\lambda x_{n}-(1-\lambda) W x_{n}\right\| \\
& \leq\left\|x_{n}-S_{n} x_{n}\right\|+\left|\beta_{n}-\lambda\right|\left\|x_{n}-W x_{n}\right\|+\left(1-\beta_{n}\right)\left\|W_{n} x_{n}-W x_{n}\right\| .
\end{aligned}
$$

It follows from (3.13), (3.15) and (3.16) that

$$
\lim _{n \rightarrow \infty}\left\|x_{n}-S x_{n}\right\|=0
$$

Now we claim that $\lim \sup _{n \rightarrow \infty}\left\langle(B-\gamma f) q, q-x_{n}\right\rangle \leq 0$, where $q=\lim _{t \rightarrow 0} x_{t}$ with $x_{t}$ being the fixed point of the contraction $\Psi_{n}$ on $H$ defined by

$$
\Psi_{n} x=t \gamma f(x)+(I-t B) S_{n} T_{r_{n}}\left(I-r_{n} A\right) x, \quad \forall x \in H, n \in N,
$$

where $t \in(0,1)$. Indeed, by Lemma 2.1 and 2.3 , we have

$$
\begin{aligned}
\left\|\Psi_{n} x-\Psi_{n} y\right\| & \leq t \gamma\|f(x)-f(y)\|+(1-t \bar{\gamma})\left\|S_{n} T_{r_{n}}\left(I-r_{n} A\right) x-S_{n} T_{r_{n}}\left(I-r_{n} A\right) y\right\| \\
& \leq t \gamma \rho\|x-y\|+(1-t \bar{\gamma})\left\|T_{r_{n}}\left(I-r_{n} A\right) x-T_{r_{n}}\left(I-r_{n} A\right) y\right\| \\
& \leq t \gamma \rho\|x-y\|+(1-t \bar{\gamma})\|x-y\| \\
& =[1-(\bar{\gamma}-\gamma \rho) t]\|x-y\|,
\end{aligned}
$$

for all $x, y \in H$. Since $0<1-(\bar{\gamma}-\gamma \rho) t<1$, it follows that $\Psi_{n}$ is a contraction. Therefore, by the Banach contraction principle, $\Psi_{n}$ has a unique fixed point $x_{t} \in H$ such that

$$
x_{t}=t \gamma f\left(x_{t}\right)+(I-t B) S_{n} T_{r_{n}}\left(I-r_{n} A\right) x_{t} .
$$


By Lemma 2.2 and (3.10), we have

$$
\begin{aligned}
\left\|x_{t}-x_{n}\right\|^{2}= & \left\|(I-t B)\left[S_{n} T_{r_{n}}\left(I-r_{n} A\right) x_{t}-x_{n}\right]+t\left[\gamma f\left(x_{t}\right)-B x_{n}\right]\right\|^{2} \\
\leq & (1-\bar{\gamma} t)^{2}\left\|S_{n} T_{r_{n}}\left(I-r_{n} A\right) x_{t}-x_{n}\right\|^{2}+2 t\left\langle\gamma f\left(x_{t}\right)-B x_{n}, x_{t}-x_{n}\right\rangle \\
= & (1-\bar{\gamma} t)^{2}\left\|S_{n} T_{r_{n}}\left(I-r_{n} A\right) x_{t}-S_{n} T_{r_{n}}\left(I-r_{n} A\right) x_{n}+S_{n} T_{r_{n}}\left(I-r_{n} A\right) x_{n}-x_{n}\right\|^{2} \\
& +2 t\left\langle\gamma f\left(x_{t}\right)-B x_{n}, x_{t}-x_{n}\right\rangle \\
\leq & (1-\bar{\gamma} t)^{2}\left[\left\|x_{t}-x_{n}\right\|+\left\|y_{n}-x_{n}\right\|\right]^{2}+2 t\left\langle\gamma f\left(x_{t}\right)-B x_{n}, x_{t}-x_{n}\right\rangle \\
\leq & (1-\bar{\gamma} t)^{2}\left\|x_{t}-x_{n}\right\|^{2}+\psi_{n}(t)+2 t\left\langle\gamma f\left(x_{t}\right)-B x_{t}, x_{t}-x_{n}\right\rangle \\
& +2 t\left\langle B x_{t}-B x_{n}, x_{t}-x_{n}\right\rangle
\end{aligned}
$$

where $\psi_{n}(t)=(1-\bar{\gamma} t)^{2}\left(2\left\|x_{t}-x_{n}\right\|+\left\|y_{n}-x_{n}\right\|\right)\left\|y_{n}-x_{n}\right\| \rightarrow 0$ as $n \rightarrow \infty$. Observe $B$ is strongly positive, we obtain

$$
\left\langle B x_{t}-B x_{n}, x_{t}-x_{n}\right\rangle=\left\langle B\left(x_{t}-x_{n}\right), x_{t}-x_{n}\right\rangle \geq \bar{\gamma}\left\|x_{t}-x_{n}\right\|^{2}
$$

Combining (3.18) and (3.19), we have

$$
\begin{aligned}
2 t\left\langle B x_{t}-\gamma f\left(x_{t}\right), x_{t}-x_{n}\right\rangle \leq & \left(\bar{\gamma}^{2} t^{2}-2 \bar{\gamma} t\right)\left\|x_{t}-x_{n}\right\|^{2}+\psi_{n}(t)+2 t\left\langle B x_{t}-B x_{n}, x_{t}-x_{n}\right\rangle \\
\leq & \left(\bar{\gamma} t^{2}-2 t\right)\left\langle B\left(x_{t}-x_{n}\right), x_{t}-x_{n}\right\rangle+\psi_{n}(t) \\
& +2 t\left\langle B x_{t}-B x_{n}, x_{t}-x_{n}\right\rangle \\
= & \bar{\gamma} t^{2}\left\langle B x_{t}-B x_{n}, x_{t}-x_{n}\right\rangle+\psi_{n}(t) .
\end{aligned}
$$

It follows that

$$
\left\langle B x_{t}-\gamma f\left(x_{t}\right), x_{t}-x_{n}\right\rangle \leq \frac{\bar{\gamma} t}{2}\left\langle B x_{t}-B x_{n}, x_{t}-x_{n}\right\rangle+\frac{1}{2 t} \psi_{n}(t)
$$

Let $n \rightarrow \infty$ in (3.20) and note that $\psi_{n}(t) \rightarrow 0$ as $n \rightarrow \infty$ yields

$$
\limsup _{n \rightarrow \infty}\left\langle B x_{t}-\gamma f\left(x_{t}\right), x_{t}-x_{n}\right\rangle \leq \frac{t}{2} M_{4}
$$

where $M_{4}$ is an appropriate positive constant such that $M_{4} \geq \bar{\gamma}\left\langle B x_{t}-B x_{n}, x_{t}-x_{n}\right\rangle$ for all $t \in(0,1)$ and $n \geq 1$. Taking $t \rightarrow 0$ from (3.21), we have

$$
\limsup _{t \rightarrow 0} \limsup _{n \rightarrow \infty}\left\langle B x_{t}-\gamma f\left(x_{t}\right), x_{t}-x_{n}\right\rangle \leq 0
$$

On the other hand, we have

$$
\begin{aligned}
\left\langle\gamma f(q)-B q, x_{n}-q\right\rangle= & \left\langle\gamma f(q)-B q, x_{n}-q\right\rangle-\left\langle\gamma f(q)-B q, x_{n}-x_{t}\right\rangle+\left\langle\gamma f(q)-B q, x_{n}-x_{t}\right\rangle \\
& -\left\langle\gamma f(q)-B x_{t}, x_{n}-x_{t}\right\rangle+\left\langle\gamma f(q)-B x_{t}, x_{n}-x_{t}\right\rangle \\
& -\left\langle\gamma f\left(x_{t}\right)-B x_{t}, x_{n}-x_{t}\right\rangle+\left\langle\gamma f\left(x_{t}\right)-B x_{t}, x_{n}-x_{t}\right\rangle .
\end{aligned}
$$


It follows that

$$
\begin{aligned}
& \limsup _{n \rightarrow \infty}\left\langle\gamma f(q)-B q, x_{n}-q\right\rangle \\
& \leq\|\gamma f(q)-B q\|\left\|x_{t}-q\right\|+\|B\|\left\|x_{t}-q\right\| \lim _{n \rightarrow \infty}\left\|x_{n}-x_{t}\right\| \\
& \quad+\gamma \rho\left\|x_{t}-q\right\| \lim _{n \rightarrow \infty}\left\|x_{n}-x_{t}\right\|+\limsup _{n \rightarrow \infty}\left\langle\gamma f\left(x_{t}\right)-B x_{t}, x_{n}-x_{t}\right\rangle .
\end{aligned}
$$

Therefore, from (3.22) and $\lim _{t \rightarrow 0} x_{t}=q$, we have

$$
\begin{aligned}
\limsup _{n \rightarrow \infty}\left\langle\gamma f(q)-B q, x_{n}-q\right\rangle= & \limsup _{t \rightarrow 0} \limsup _{n \rightarrow \infty}\left\langle\gamma f(q)-B q, x_{n}-q\right\rangle \\
\leq & \limsup _{t \rightarrow 0}\|\gamma f(q)-B q\|\left\|x_{t}-q\right\| \\
& +\limsup _{t \rightarrow 0}\|B\|\left\|x_{t}-q\right\| \lim _{n \rightarrow \infty}\left\|x_{n}-x_{t}\right\| \\
& +\limsup _{t \rightarrow 0} \gamma \rho x_{t}-q\left\|\lim _{n \rightarrow \infty}\right\| x_{n}-x_{t} \| \\
& +\limsup _{t \rightarrow 0} \limsup _{n \rightarrow \infty}\left\langle\gamma f\left(x_{t}\right)-B x_{t}, x_{n}-x_{t}\right\rangle \\
\leq & 0 .
\end{aligned}
$$

Finally, we prove that $x_{n} \rightarrow q$ as $n \rightarrow \infty$. From (1.4) and (3.2) again, we have

$$
\begin{aligned}
\left\|x_{n+1}-q\right\|^{2}= & \left\langle\alpha_{n} \gamma f\left(x_{n}\right)+\left(I-\alpha_{n} B\right) y_{n}-q, x_{n+1}-q\right\rangle \\
= & \alpha_{n}\left\langle\gamma f\left(x_{n}\right)-B q, x_{n+1}-q\right\rangle+\left\langle\left(I-\alpha_{n} B\right)\left(y_{n}-q\right), x_{n+1}-q\right\rangle \\
\leq & \alpha_{n} \gamma\left\langle f\left(x_{n}\right)-f(q), x_{n+1}-q\right\rangle+\alpha_{n}\left\langle\gamma f(q)-B q, x_{n+1}-q\right\rangle \\
& +\left(1-\alpha_{n} \bar{\gamma}\right)\left\|y_{n}-q\right\|\left\|x_{n+1}-q\right\| \\
\leq & \alpha_{n} \gamma \rho\left\|x_{n}-q\right\|\left\|x_{n+1}-q\right\|+\alpha_{n}\left\langle\gamma f(q)-B q, x_{n+1}-q\right\rangle \\
& +\left(1-\alpha_{n} \bar{\gamma}\right)\left\|x_{n}-q\right\|\left\|x_{n+1}-q\right\| \\
= & {\left[1-(\bar{\gamma}-\gamma \rho) \alpha_{n}\right]\left\|x_{n}-q\right\|\left\|x_{n+1}-q\right\|+\alpha_{n}\left\langle\gamma f(q)-B q, x_{n+1}-q\right\rangle } \\
\leq & \frac{1-(\bar{\gamma}-\gamma \rho) \alpha_{n}}{2}\left(\left\|x_{n}-q\right\|^{2}+\left\|x_{n+1}-q\right\|^{2}\right)+\alpha_{n}\left\langle\gamma f(q)-B q, x_{n+1}-q\right\rangle \\
\leq & \frac{1-(\bar{\gamma}-\gamma \rho) \alpha_{n}}{2}\left\|x_{n}-q\right\|^{2}+\frac{1}{2}\left\|x_{n+1}-q\right\|^{2}+\alpha_{n}\left\langle\gamma f(q)-B q, x_{n+1}-q\right\rangle .
\end{aligned}
$$

It follows that

$$
\left\|x_{n+1}-q\right\|^{2} \leq\left[1-(\bar{\gamma}-\gamma \rho) \alpha_{n}\right]\left\|x_{n}-q\right\|^{2}+2 \alpha_{n}\left\langle\gamma f(q)-B q, x_{n+1}-q\right\rangle .
$$

From $0<\gamma<\frac{\bar{\gamma}}{\rho}$, condition (i) and (3.23), we can arrive at the desired conclusion $\lim _{n \rightarrow \infty}\left\|x_{n}-q\right\|=0$ by Lemma 2.6. This completes the proof.

Theorem 3.2 Let $K$ be a nonempty closed convex subset of the Hilbert space $H$ and $F: K \times K \rightarrow \mathbb{R}$ be a bi-function satisfying (A1)-(A4). Let $A$ be an $\alpha$-inverse strongly monotone mapping, $f \in \Pi_{K}$ with a coefficient $\rho \in(0,1)$ and $B$ be a strongly positive bounded linear operator on $H$ with $\bar{\gamma}>0$ and $0<\gamma<\frac{\bar{\gamma}}{\rho}$. Let $T: K \rightarrow H$ be a $k$-strict pseudo-contraction 
such that $\mathcal{F}=F(T) \cap E P(F, A) \neq \phi$. Let $\left\{x_{n}\right\}$ be a sequence generated by $x_{0} \in K$ in the following manner:

$$
\left\{\begin{array}{l}
F\left(u_{n}, y\right)+\left\langle A x_{n}, y-u_{n}\right\rangle+\frac{1}{r}\left\langle y-u_{n}, u_{n}-x_{n}\right\rangle \geq 0, \quad \forall y \in K, \\
y_{n}=\beta_{n} u_{n}+\left(1-\beta_{n}\right) T u_{n}, \\
x_{n+1}=\alpha_{n} \gamma f\left(x_{n}\right)+\left(I-\alpha_{n} B\right) y_{n}, \quad n \geq 1,
\end{array}\right.
$$

where $\left\{\alpha_{n}\right\}$ and $\left\{\beta_{n}\right\}$ are two sequences in $(0,1)$, constant $r \in(0,2 \alpha)$. If the following control conditions are satisfied:

(i) $\lim _{n \rightarrow \infty} \alpha_{n}=0, \sum_{n=1}^{\infty} \alpha_{n}=\infty$ and $\sum_{n=1}^{\infty}\left|\alpha_{n}-\alpha_{n-1}\right|<\infty$;

(ii) $k \leq \beta_{n} \leq \lambda<1, \lim _{n \rightarrow \infty} \beta_{n}=\lambda$ and $\sum_{n=1}^{\infty}\left|\beta_{n}-\beta_{n-1}\right|<\infty$.

Then the sequence $\left\{x_{n}\right\}$ converges strongly to $q \in \mathcal{F}$, which solves the variational inequality

$$
\langle(B-\gamma f) q, q-p\rangle \leq 0, \quad \forall p \in \mathcal{F} .
$$

Proof Putting $r_{n}=r$ and $N=1$, i.e., $W_{n}=T$, the desired conclusion follows immediately from Theorem 3.1. This completes the proof.

Theorem 3.3 Let $K$ be a nonempty closed convex subset of the Hilbert space $H$ and $F$ : $K \times K \rightarrow \mathbb{R}$ be a bi-function satisfying (A1)-(A4). Let $f \in \Pi_{K}$ with a coefficient $\rho \in(0,1)$ and $B$ be a strongly positive bounded linear operator on $H$ with $\bar{\gamma}>0$ and $0<\gamma<\frac{\bar{\gamma}}{\rho}$. Let $T: K \rightarrow H$ be a $k$-strict pseudo-contraction such that $\mathcal{F}=F(T) \cap E P(F) \neq \phi$. Let $\left\{x_{n}\right\}$ be a sequence generated by $x_{0} \in K$ in the following manner:

$$
\left\{\begin{array}{l}
F\left(u_{n}, y\right)+\frac{1}{r_{n}}\left\langle y-u_{n}, u_{n}-x_{n}\right\rangle \geq 0, \quad \forall y \in K, \\
y_{n}=\beta_{n} u_{n}+\left(1-\beta_{n}\right) T u_{n}, \\
x_{n+1}=\alpha_{n} \gamma f\left(x_{n}\right)+\left(I-\alpha_{n} B\right) y_{n}, \quad n \geq 1,
\end{array}\right.
$$

where $\left\{\alpha_{n}\right\}$ and $\left\{\beta_{n}\right\}$ are two sequences in $(0,1)$, sequence $\left\{r_{n}\right\} \subset(0,2 \alpha)$. If the following control conditions are satisfied:

(i) $\lim _{n \rightarrow \infty} \alpha_{n}=0, \sum_{n=1}^{\infty} \alpha_{n}=\infty$ and $\sum_{n=1}^{\infty}\left|\alpha_{n}-\alpha_{n-1}\right|<\infty$;

(ii) $k \leq \beta_{n} \leq \lambda<1, \lim _{n \rightarrow \infty} \beta_{n}=\lambda$ and $\sum_{n=1}^{\infty}\left|\beta_{n}-\beta_{n-1}\right|<\infty$;

(iii) $\liminf _{n \rightarrow \infty} r_{n}>0$ and $\sum_{n=1}^{\infty}\left|r_{n}-r_{n-1}\right|<\infty$.

Then the sequence $\left\{x_{n}\right\}$ converges strongly to $q \in \mathcal{F}$, which solves the variational inequality

$$
\langle(B-\gamma f) q, q-p\rangle \leq 0, \quad \forall p \in \mathcal{F} .
$$

Proof Putting $N=1$ and $A=0$, i.e., the generalized equilibrium problem (1.1) reduces to the normal equilibrium problem (1.2), the desired conclusion follows immediately from Theorem 3.1. This completes the proof.

Remark 3.1 Theorem 3.1 and 3.2 improve and extend the main results of Takahashi and Takahashi [18] and Qin et al. [21] in different directions.

Remark 3.2 Theorem 3.3 is mainly due to Liu [16], which improves and extends the main results of Takahashi and Takahashi [12]. 
Remark 3.3 If $F=A=0$ and $u_{n}=x_{n}$, then the algorithm (1.4) reduces to approximate the fixed point of $k$-strict pseudo-contractions, which includes the general iterative method of Marino and $\mathrm{Xu}[8]$ and the parallel algorithm of Acedo and $\mathrm{Xu}$ [20] as special cases.

\section{Competing interests}

The authors declare that they have no competing interests.

\section{Authors' contributions}

Wen, DJ carried out the primary studies for the generalized equilibrium problems and fixed point problems of $k$-strict pseudo-contractions, participated in the design of iterative methods and drafted the manuscript. Chen YA participated in the convergence analysis and coordination. All authors read and approved the final manuscript.

\section{Acknowledgements}

Supported by the National Science Foundation of China (11001287), Natural Science Foundation Project of Changging (CSTC 2012jjA00039) and Science and Technology Research Project of Chongqing Municipal Education Commission (KJ 110701).

Received: 18 December 2011 Accepted: 11 July 2012 Published: 27 July 2012

\section{References}

1. Blum, E, Oettli, W: From optimization and variational inequalities to equilibrium problems. Math. Stud. $63,123-145$ (1994)

2. Moudafi, A, Thera, M: Proximal and dynamical approaches to equilibrium problems. In: III-posed Variational Problems and Regularization Techniques. Lecture Notes in Economics and Mathematical Systems, vol. 477, pp. 187-201. Springer, Berlin (1999)

3. Combettes, PL, Hirstoaga, A: Equilibrium programming in Hilbert spaces. J. Nonlinear Convex Anal. 6, 117-136 (2005)

4. Browder, FE: Convergence of approximants to fixed points of nonexpansive nonlinear mappings in Banach spaces. Arch. Ration. Mech. Anal. 24, 82-90 (1967)

5. Browder, FE, Petryshyn, WV: Construction of fixed points of nonlinear mappings in Hilbert space. J. Math. Anal. Appl. 20, 197-228 (1967)

6. Scherzer, O: Convergence criteria of iterative methods based on Landweber iteration for solving nonlinear problems. J. Math. Anal. Appl. 194, 911-933 (1991)

7. Plubtieng, S, Punpaeng, R: A general iterative method for equilibrium problems and fixed point problems in Hilbert spaces. J. Math. Anal. Appl. 336, 455-469 (2007)

8. Marino, G, Xu, HK: A general iterative method for nonexpansive mappings in Hilbert spaces. J. Math. Anal. Appl. 318 43-52 (2006)

9. Ceng, LC, Yao, JC: Hybrid viscosity approximation schemes for equilibrium problems and fixed point problems of infinitely many nonexpansive mappings. Appl. Math. Comput. 198, 729-741 (2008)

10. Zhou, $\mathrm{H}$ : Convergence theorems of fixed points for $k$-strict pseudo-contractions in Hilbert spaces. Nonlinear Anal. 69 456-462 (2008)

11. Marino, G, Xu, HK: Weak and strong convergence theorems for k-strict pseudo-contractions in Hilbert spaces. J. Math. Anal. Appl. 329, 336-349 (2007)

12. Takahashi, S, Takahashi, W: Viscosity approximation methods for equilibrium problems and fixed point problems in Hilbert spaces. J. Math. Anal. Appl. 331, 506-515 (2007)

13. Plubtieng, S, Punpaeng, R: A new iterative method for equilibrium problems and fixed point problems of nonexpansive mappings and monotone mappings. Appl. Math. Comput. 197, 548-558 (2008)

14. Chang, SS, Joseph Lee, HW, Chan, CK: A new method for solving equilibrium problem fixed point problem and variational inequality problem with application to optimization. Nonlinear Anal. 70, 3307-3319 (2009)

15. Ceng, LC, Al-Homidan, S, Ansari, QH, Yao, JC: An iterative scheme for equilibrium problems and fixed point problems of strict pseudo-contraction mappings. J. Comput. Appl. Math. 223, 967-974 (2009)

16. Liu, Y: A general iterative method for equilibrium problems and strict pseudo-contractions in Hilbert spaces. Nonlinear Anal. 71, 4852-4861 (2009)

17. Qin, $X$, Cho, YJ, Kang, SM: Convergence theorems of common elements for equilibrium problems and fixed point problems in Banach spaces. J. Comput. Appl. Math. 225, 20-30 (2009)

18. Takahashi, S, Takahashi, W: Strong convergence theorem for a generalized equilibrium problem and a nonexpansive mapping in a Hilbert space. Nonlinear Anal. 69, 1025-1033 (2008)

19. Xu, HK: An iterative approach to quadratic optimization. J. Optim. Theory Appl. 116, 659-678 (2003)

20. Acedo, GL, Xu, HK: Iteration methods for strict pseudo-contractions in Hilbert spaces. Nonlinear Anal. 67, 2258-2271 (2007)

21. Qin, X, Cho, YJ, Kang, SM: Viscosity approximation methods for generalized equilibrium problems and fixed point problems with applications. Nonlinear Anal. 72, 99-112 (2010)

22. Wen, DJ: Projection methods for a generalized system of nonconvex variational inequalities with different nonlinear operators. Nonlinear Anal. 73, 2292-2297 (2010)

23. Chang, SS, Chan, CK, Joseph Lee, HW Yang, L: A system of mixed equilibrium problems, fixed point problems of strictly pseudo-contractive mappings and nonexpansive semi-groups. Appl. Math. Comput. 216, 51-60 (2010)

24. Kang, SM, Cho, SY, Liu, Z: Convergence of iterative sequences for generalized equilibrium problems involving inverse-strongly monotone mappings. J. Inequal. Appl. 2010, 827082 (2010) 
25. Wen, DJ: Strong convergence theorems for equilibrium problems and $k$-strict pseudocontractions in Hilbert spaces. Abstr. Appl. Anal. (2011). doi:10.1155/2011/276874

26. Cho, SY, Kang, SM: Approximation of fixed points of pseudocontraction semigroups based on a viscosity iterative process. Appl. Math. Lett. 24, 224-228 (2011)

27. Ye, J, Huang, J: Strong convergence theorems for fixed point problems and generalized equilibrium problems of three relatively quasi-nonexpansive mappings in Banach spaces. J. Math. Comput. Sci. 1, 1-18 (2011)

doi:10.1186/1687-1812-2012-125

Cite this article as: Wen and Chen: General iterative methods for generalized equilibrium problems and fixed point problems of $k$-strict pseudo-contractions. Fixed Point Theory and Applications 2012 2012:125.

Submit your manuscript to a SpringerOpen ${ }^{\circ}$ journal and benefit from:

- Convenient online submission

- Rigorous peer review

Immediate publication on acceptance

- Open access: articles freely available online

- High visibility within the field

- Retaining the copyright to your article

Submit your next manuscript at $>$ springeropen.com 